\title{
The dual behavior of PCSK9 in the regulation of apoptosis is crucial in Alzheimer's disease progression (Review)
}

\author{
QI WU ${ }^{1 *}$, ZHI-HAN TANG ${ }^{1,2^{*}}$, JUAN PENG $^{1}$, LING LIAO $^{1}$, LI-HONG PAN ${ }^{1}$, \\ CHUN-YAN WU ${ }^{1}$, ZHI-SHENG JIANG ${ }^{1}$, GUI-XUE WANG ${ }^{1,2}$ and LU-SHAN LIU ${ }^{1}$ \\ ${ }^{1}$ Institute of Cardiovascular Disease, Key Laboratory for Arteriosclerology of Hunan Province, University of South \\ China, Hengyang, Hunan 421001; ${ }^{2}$ Bioengineering College of Chongqing University, Chongqing 400044, P.R. China
}

Received September 05, 2013; Accepted November 28, 2013

DOI: $10.3892 /$ br.2013.213

\begin{abstract}
Neuronal apoptosis is crucial in neurodegenerative diseases. However, a lower apoptotic rate of nerve cells is detected in the brain compared to that in other organs in neurodegenerative patients or in animal models, suggesting that neuronal apoptosis induced by any type of risk factors is intricately regulated. Human and animal studies demonstrated that a high concentration of oxidized LDL (ox-LDL) in the brain, which is associated with hyperlipidemia, is one of the key apoptosis inducers in neurodegenerative diseases. However, the mechanism underlying the ox-LDL-mediated regulation of neuronal apoptosis has not been fully elucidated. Recently, we investigated proprotein convertase subtilisin/kexin type 9 (PCSK9), a striking gene involved in lipid metabolism that exhibits a positive correlation with macrophage and endothelial cell apoptosis induced by ox-LDL. Moreover, PCSK 9 may degrade $\beta$-site amyloid precursor protein-cleaving enzyme 1 (BACE1), the key enzyme cleaving amyloid precursor protein (APP) to generate amyloid $\beta$ peptide $(A \beta)$. A $\beta$ is another key apoptosis inducer in neurodegenerative diseases. Our findings indicated that PCSK9 may be upregulated by the high levels of ox-LDL in the brain associated with hyperlipidemia and promote neuronal apoptosis through the NF- $\kappa \mathrm{B}-\mathrm{B}-\mathrm{cell}$ lymphoma 2 (Bcl-2)/Bax-caspase 9-caspase 3 signaling pathways. Moreover, increased PCSK9 levels may inhibit
\end{abstract}

Correspondence to: Professor Lu-Shan Liu, Institute of Cardiovascular Disease, Key Laboratory for Arteriosclerology of Hunan Province, University of South China, 28 West Changsheng Road, Zhengxiang District, Hengyang, Hunan 421001, P.R. China E-mail: liuls2000@163.com

Professor Gui-Xue Wang, Bioengineering College of Chongqing University, 174 Shazhengjie Street, Shapingba District, Chongqing 400044, P.R. China

E-mail: guixue_wang@126.com

"Contributed equally

Key words: proprotein convertase subtilisin/kexin type 9, apoptosis, Alzheimer's disease, oxidized low-density lipoprotein, $\beta$-site amyloid precursor protein-cleaving enzyme 1 , amyloid $\beta$ peptide the APP/A $\beta$ metabolic pathway and reduce $A \beta$ generation by degrading BACE1, thereby decreasing $\mathrm{A} \beta$-induced neuronal apoptosis. The dual regulation mechanism of PCSK9 on apoptosis maintains neuronal apoptosis induced by risk factors at low levels.

\section{Contents}

1. Introduction

2. Neuronal apoptosis rate is lower compared to that in other cell types

3. PCSK9 promotes neuronal apoptosis

4. PCSK9 inhibits neuronal apoptosis

5. Inconsistencies and future directions

\section{Introduction}

In 2003, proprotein convertase subtilisin/kexin type 9(PCSK9) was identified by the bioinformatics method and DNA microarray technology $(1,2)$. PCSK9 is a member of the subtilisin family of proprotein convertases and is involved in the degradation of low-density lipoprotein receptor (LDLR) in the liver (3). Gain- and loss-of-function mutations of PCSK9 may result in hyper- and hypocholesterolemia, respectively, thereby affecting liver LDLR degradation and blood lipid levels. Cohen et al (4) reported that African-Americans harbouring nonsense mutations in PCSK9 exhibited a $28 \%$ decrease in LDL cholesterol levels and a $88 \%$ lower risk of coronary heart disease (CHD), whereas Caucasians with less severe mutations in PCSK9 exhibited a $15 \%$ decrease in LDL levels and a $47 \%$ lower risk of CHD. Surprisingly, the LDL-lowering mutations of PCSK9 were shown to exert a protective effect in CHD. A previous phase II clinical trial of a monoclonal antibody to PCSK9 demonstrated that patients with severe heterozygous familial hypercholesterolemia on high-dose statins who were injected with anti-PCSK9 monoclonal antibody exhibited maximum LDL-C levels (5). The results, however, depend on the phase III side effects of the anti-PCSK9 monoclonal antibody (6). If proven efficient, the combination of anti-PCSK9 anibodies with statins may become a potent lipid-lowering 
and cardioprotective treatment that should be used prior to the advancement of atherosclerosis, since the effects of lowering LDL levels do not depend solely on the extent, but also on the duration of the decrease (7). The extensive investigation regarding PCSK9 revealed novel functions, in addition to those in lipid metabolism, including cell apoptosis, inflammatory response, neuronal development and tumor metastasis (8-11).

Neurodegeneration is a progressive loss of neurons and synapses in the cerebral cortex and certain subcortical regions. A number of neurodegenerative disorders (ND), such as Parkinson's disease, Alzheimer's disease (AD) and Huntington's disease, occur as a result of neurodegenerative processes. AD is the major form of ND and is characterized by senile plaques and neurofibrillary tangles in the brain. Excessive generation and accumulation of amyloid $\beta$ peptide $(A \beta)$ in the brain is an important factor implicated in AD. Neuronal apoptosis is also a primary mechanism underlying AD pathogenesis. Table I shows the numerous pro- and anti-apoptotic factors that may mediate neuronal apoptosis. Although several treatments are currently available to mitigate the progression of the disease, there is no established medical method to definitively prevent the progression of $\mathrm{AD}$; the loss of neurons is permanent, due to their lack of proliferative ability (12).

\section{Neuronal apoptosis rate is lower compared to that in other cell types}

Using New Zealand rabbit models on a high-fat diet for 7 months, our research demonstrated that hyperlipidemia may induce neuronal apoptosis. Higher rates of apoptosis in the brain were observed in the high-fat diet group, compared to those in the normal cholesterol group (0.83 vs. $0.30 \%$, unpublished data). A study by Cunningham et al (13) also reported that the apoptosis rate in brain nerve cells induced by endotoxins in neurodegenerative mouse models is lower, which is in accordance with the findings of our study. However, compared to other systems, hyperlipidemia is associated with significantly lower rates of neuronal apoptosis in the brain. The apoptosis rate of smooth muscle cells in atherosclerotic lesions was shown to be $11 \pm 7.8 \%$ in miniature pigs on a high-fat diet for 37 weeks (14). Nematbakhsh et al (15) observed an endothelial cell apoptosis rate of $8 \%$ in hyperlipidemic rabbits. However, the reason for the significant difference in the apoptosis rate of nerve cells and other cells exposed to hyperlipidemia has not been determined. It was hypothesized that the presence of the blood-brain barrier (BBB) was a possible explanation. However, the oxidized LDL (ox-LDL) content was found to be increased in the brain and in the blood of AD patients (16), indicating that $\mathrm{BBB}$ does not play a major role under hyperlipidemic conditions. Martins et al (17) proposed two mechanisms that may underlie the increase in the lipid content of the brain during hyperlipidemia. The first mechanism is BBB damage. Ox-LDL may damage the BBB by inducing endothelial cell apoptosis, which increases the permeability of the BBB membrane, allowing lipids to cross the BBB into the brain. The second mechanism involves necessary components involved in lipid synthesis, such as unsaturated fatty acids, crossing the BBB, thereby increasing endogenous lipid synthesis in the brain. The brain lipidogram is significantly affected in hyperlipidemia. However, the effect of the BBB
Table I. Pro- and anti-apoptotic factors in neurons.

\begin{tabular}{lc} 
Pro-apoptotic (Refs.) & Anti-apoptotic (Refs.) \\
\hline NF- $\mathrm{B}(30)$ & Bcl-2 $(31,32)$ \\
Bax $(32-35)$ & Bcl-x(L) $(31,32,34)$ \\
Bak $(34)$ & IAPs $(36,37)$ \\
Bad $(32,38)$ & CrmA (39) \\
Bid $(32)$ & FLIPs $(40)$ \\
Caspase-3, $-6,-8,-9(35,41-43)$ & IGF-1 (44) \\
TNF- $\alpha(35)$ & TGF- $\beta(38)$ \\
p53 $(33)$ & p35 $(45)$ \\
Fas/FasL $(35)$ & ARC (46) \\
Cytochrome $c(41)$ & VEGF (47) \\
AIF (48) & \\
\hline
\end{tabular}

TNF, tumor necrosis factor; AIF, apoptosis-inducing factor; Bcl, B-cell lymphoma; IAPs, inhibitors of apoptosis; Crm, cytokine response modifier; FLIPs, FADD-like interleukin-1 $\beta$-converting enzyme (FLICE)-inhibitory proteins; TGF, transforming growth factor; ARC, apoptosis repressor with caspase recruitment domain; VEGF, vascular endothelial growth factor.

on the apoptosis rate is minimal. We further hypothesized that a pathway inhibiting neuronal apoptosis may exist in the process of hyperlipidemia, along with an additional pathway inducing brain nerve cell apoptosis; however, further investigation is required to identify the common regulation of the two diametrically opposed pathways.

\section{PCSK9 promotes neuronal apoptosis}

In this study, we mainly focused on the association between cell apoptosis and PCSK9. The significant correlations between PCSK9 and cell apoptosis are as follows: i) Wu et al (8) reported that ox-LDL may upregulate PCSK9 expression in human umbilical vein endothelial cells (HUVECs), whereas PCSK9 siRNA inhibits HUVEC apoptosis induced by ox-LDL through the $\mathrm{Bcl} / \mathrm{Bax}$-caspase 9-caspase 3 pathway; and ii) previous studies suggested that PCSK9 may be associated with neuronal apoptosis $(18,19)$. Cameron et al $(20)$ reported that berberine may decrease PCSK9 expression, whereas $\mathrm{Ji}$ and Shen (21) indicated that berberine exerts a potentially preventive effect on AD through a variety of mechanisms. We therefore considered the potential involvement of PCSK9 in the effect of berberine against AD. The link between PCSK9 and apoptosis was previously investigated. PCSK9 may regulate the expression of inflammatory factors induced by ox-LDL through NF- $\mathrm{B}(9)$, whereas the $\mathrm{NF}-\kappa \mathrm{B} / \mathrm{B}$-cell lymphoma 2 (Bcl-2) signaling pathway is crucial in mediating apoptosis. The effect of PCSK9 in cerebellar granule neuron apoptosis is reflected by the fact that the effects of wild-type and mutant PCSK9 on apoptosis are partially reversed by BAF, a caspase inhibitor. Moreover, apoptotic mediators, such as caspase 3 and death receptor 6, may be involved in neuronal apoptotic signaling induced by PCSK9 (19). A recent study demonstrated that PCSK9 promotes neuronal apoptosis through the decreae 


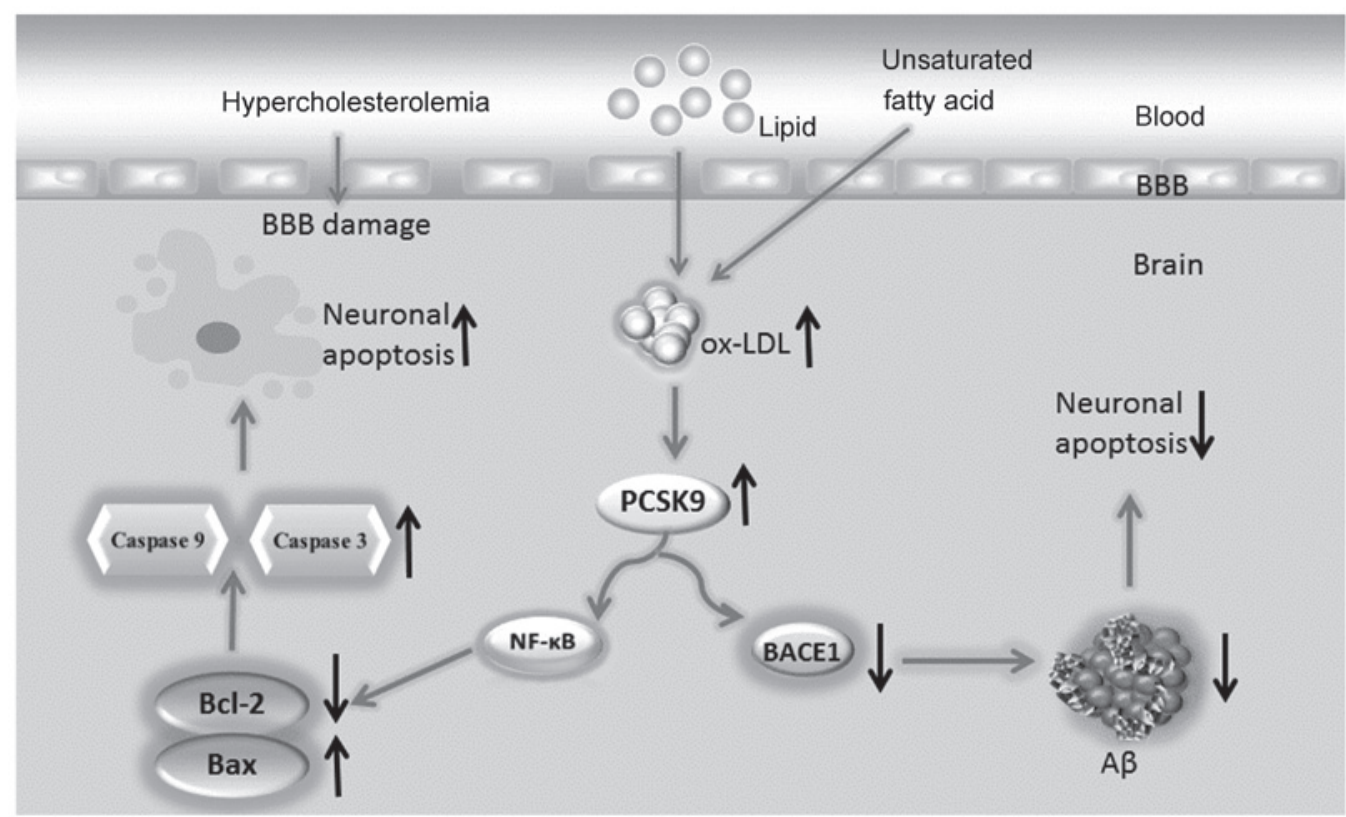

Figure 1. Dual regulatory effect of proprotein convertase subtilisin/kexin type 9 (PCSK9) on neuronal apoptosis. Hyperlipidemia increases the lipid content in the brain, particularly oxidized LDL (ox-LDL). High ox-LDL levels may upregulate the PCSK9 expression in neurons. Neuronal apoptosis is induced through the NF-кB-Bcl-2/Bax-caspase 9-caspase 3 signaling pathway when PCSK9 expression increases. Additionally, through BACE1 degradation, PCSK9 may reduce amyloid $\beta$ peptide $(A \beta)$ generation by inhibiting the amyloid precursor protein/A $\beta$ metabolic pathway, thereby decreasing neuronal apoptosis induced by $A \beta$.

of apolipoprotein E receptor 2 levels and deregulation of anti-apoptotic signaling pathways (22). However, further investigations are required to identify the precise PCSK9-related mechanism leading to neuronal apoptosis.

\section{PCSK9 inhibits neuronal apoptosis}

Although PCSK9 is also referred to as neural apoptosis-regulated convertase 1 , certain studies failed to confirm its pro-apoptotic effect. Ranheim et al (23) demonstrated that PCSK9 significantly downregulated the poly(ADP-ribose) polymerase (PARP) family in HepG2 cells through microarray analysis of D374Y-PCSK9, a gain-of-function mutant. B16F1 melanoma cells were injected in PCSK9-/- mice to induce liver metastasis. The findings revealed an increased rate of apoptosis in the liver stroma and metastases. Furthermore, the pro-apoptotic factor tumor necrosis factor- $\alpha$ levels were increased and the anti-apoptotic factor Bcl-2 levels were decreased (10), confirming the anti-apoptotic function of PCSK9. However, the precise mechanism through which PCSK9 inhibits neuronal apoptosis has not been fully elucidated.

The following pathways are involved in amyloid precursor protein (APP) metabolism: i) APP is cleaved by $\alpha$-secretase to generate soluble APP- $\alpha$ and C83; ii) APP is cleaved by the $\beta$-site APP-cleaving enzyme 1 (BACE1) and $\gamma$-secretase to generate insoluble $A \beta$. The former is generally considered to be the major metabolic pathway. Under pathological conditions, the generation of numerous $\mathrm{A} \beta$ peptides was suggested to cause AD (24). The BACE1 level is increased in the majority of sporadic types of AD. Consistent with the increase in $\mathrm{BACE} 1$, higher concentrations of $\mathrm{A} \beta$ may induce neuronal apoptosis and lead to AD.

Jonas et al (25) and and Ko and Puglielli (26) observed that the levels of BACE1 and $A \beta$ were increased in the brains of
PCSK9(-/-) mice, the overexpression of PCSK9 in CHO cells decreased the level of BACE1, PCSK9 siRNA increased the level of BACE1 and secreted PCSK9 may stimulate the degradation of BACE1. Those findings indicated that PCSK 9 possibly decreases $A \beta$ generation to prevent neuronal apoptosis through the degradation of BACE1 in nerve cells. However, Liu et al (27) reported that the levels of PCSK9 and BACE1 expression are not important; thus, further investigations are required to determine whether they affect BACE1 activity.

\section{Inconsistencies and future directions}

Shibata et al (28) investigated two single-nucleotide polymorphisms (SNPs) of PCSK9, namely rs11583680 and rs662145, and suggested that SNPs are not associated with AD. In addition, Reynolds et al (29) investigated the association between lipid pathway genes and AD and reported that PCSK9 is not associated with AD. However, although the above-mentioned epidemiologic studies did not lead to a definitive conclusion regarding the positive correlation between PCSK9 and AD under site selection, case scale, or other reasons, PCSK9 may still be involved in the pathogenesis of AD.

In this study, we proposed the following hypothesis: hyperlipidemia increases the lipid content, particularly ox-LDL, of the brain. The increased levels of ox-LDL may upregulate PCSK9 expression in nerve cells. Neuronal apoptosis is induced through the NF- $\mathrm{B}$-Bcl-2/Bax-caspase 9-caspase 3 signaling pathway when PCSK9 expression increases. Additionally, through the degradation of BACE1, PCSK9 decreases A $\beta$ generation via the inhibition of the APP/A $\beta$ metabolic pathway, which decreases neuronal apoptosis induced by $\mathrm{A} \beta$. Therefore, PCSK9 exerts a dual regulatory effect on neuronal apoptosis (Fig. 1), maintaining apoptosis at a low level or 
limiting its increase, leading to the slow progression of ND. The elucidation of the association among hyperlipidemia, PCSK9 expression and neuronal apoptosis requires further investigations and animal testing. Furthermore, along with the anti-PCSK9 antibody, which was proven successful in lowering lipid levels (5), drugs targeting PCSK9 are eagerly anticipated for evaluation in the treatment of $\mathrm{AD}$.

\section{Acknowledgements}

This study was supported by grants from the National Natural Science Foundation of China (81200217), the Key Project of the Educational Department of Hunan Province (10A105), the Project of the Health Department of Hunan Province (B2011-042), the Aid Program for Science and Technology Innovative Research Team in Higher Educational Institutions of Hunan Province and the National Natural Science Foundation of China (81370376).

\section{References}

1. Maxwell KN, Soccio RE, Duncan EM, Sehayek E and Breslow JL: Novel putative SREBP and LXR target genes identified by microarray analysis in liver of cholesterol-fed mice. J Lipid Res 44: 2109-2119, 2003.

2. Seidah NG, Benjannet S, Wickham L, et al: The secretory proprotein convertase neural apoptosis-regulated convertase 1 (NARC-1): liver regeneration and neuronal differentiation. Proc Natl Acad Sci USA 100: 928-933, 2003.

3. Seidah NG: PCSK9 as a therapeutic target of dyslipidemia. Expert Opin Ther Targets 13: 19-28, 2009.

4. Cohen JC, Boerwinkle E, Mosley TH Jr and Hobbs HH: Sequence variations in PCSK9, low LDL, and protection against coronary heart disease. N Engl J Med 354: 1264-1272, 2006.

5. Stein EA, Gipe D, Bergeron J, et al: Effect of a monoclonal antibody to PCSK9, REGN727/SAR236553, to reduce low-density lipoprotein cholesterol in patients with heterozygous familial hypercholesterolaemia on stable statin dose with or without ezetimibe therapy: a phase 2 randomised controlled trial. Lancet 380: 29-36, 2012.

6. Hall SS: Genetics: a gene of rare effect. Nature 496: 152-155, 2013.

7. Brown MS and Goldstein JL: Biomedicine. Lowering LDL - not only how low, but how long? Science 311: 1721-1723, 2006.

8. Wu CY, Tang ZH, Jiang L, Li XF, Jiang ZS and Liu LS: PCSK9 siRNA inhibits HUVEC apoptosis induced by ox-LDL via Bcl/Bax-caspase9-caspase3 pathway. Mol Cell Biochem 359: 347-358, 2012.

9. Tang Z, Jiang L, Peng J, et al: PCSK9 siRNA suppresses the inflammatory response induced by oxLDL through inhibition of NF- $\kappa \mathrm{B}$ activation in THP-1-derived macrophages. Int $\mathrm{J}$ Mol Med 30: 931-938, 2012.

10. Sun X, Essalmani R, Day R, Khatib AM, Seidah NG and Prat A: Proprotein convertase subtilisin/kexin type 9 deficiency reduces melanoma metastasis in liver. Neoplasia 14: 1122-1131, 2012.

11. Poirier S, Prat A, Marcinkiewicz E, et al: Implication of the proprotein convertase NARC-1/PCSK9 in the development of the nervous system. J Neurochem 98: 838-850, 2006.

12. Citron M: Alzheimer's disease: strategies for disease modification. Nat Rev Drug Discov 9: 387-398, 2010.

13. Cunningham C, Wilcockson DC, Campion S, Lunnon K and Perry VH: Central and systemic endotoxin challenges exacerbate the local inflammatory response and increase neuronal death during chronic neurodegeneration. J Neurosci 25: 9275-9284, 2005.

14. Verhamme P, Quarck R, Hao H, et al: Dietary cholesterol withdrawal reduces vascular inflammation and induces coronary plaque stabilization in miniature pigs. Cardiovasc Res 56: 135-144, 2002.

15. Nematbakhsh M, Haghjooyjavanmard S, Mahmoodi F and Monajemi AR: The prevention of endothelial dysfunction through endothelial cell apoptosis inhibition in a hypercholesterolemic rabbit model: the effect of L-arginine supplementation. Lipids Health Dis 7: 27, 2008.
16. Kankaanpaa J, Turunen SP, Moilanen V, Horkko S and Remes AM: Cerebrospinal fluid antibodies to oxidized LDL are increased in Alzheimer's disease. Neurobiol Dis 33: 467-472, 2009.

17. Martins IJ, Berger T, Sharman MJ, Verdile G, Fuller SJ and Martins RN: Cholesterol metabolism and transport in the pathogenesis of Alzheimer's disease. J Neurochem 111: 1275-1308, 2009.

18. Chiang LW, Grenier JM, Ettwiller L, et al: An orchestrated gene expression component of neuronal programmed cell death revealed by cDNA array analysis. Proc Natl Acad Sci USA 98: 2814-2819, 2001

19. Bingham B, Shen R, Kotnis S, et al: Proapoptotic effects of NARC 1 (= PCSK9), the gene encoding a novel serine proteinase. Cytometry A 69: 1123-1131, 2006.

20. Cameron J, Ranheim T, Kulseth MA, Leren TP and Berge KE: Berberine decreases PCSK9 expression in HepG2 cells. Atherosclerosis 201: 266-273, 2008.

21. Ji HF and Shen L: Berberine: a potential multipotent natural product to combat Alzheimer's disease. Molecules 16: 6732-6740, 2011

22. Kysenius K, Muggalla P, Matlik K, Arumae U and Huttunen HJ: PCSK9 regulates neuronal apoptosis by adjusting ApoER2 levels and signaling. Cell Mol Life Sci 69: 1903-1916, 2012.

23. Ranheim T, Mattingsdal M, Lindvall JM, et al: Genome-wide expression analysis of cells expressing gain of function mutant D374Y-PCSK9. J Cell Physiol 217: 459-467, 2008.

24. Di Paolo G and Kim TW: Linking lipids to Alzheimer's disease: cholesterol and beyond. Nat Rev Neurosci 12: 284-296, 2011.

25. Jonas MC, Costantini C and Puglielli L: PCSK9 is required for the disposal of non-acetylated intermediates of the nascent membrane protein BACE1. EMBO Rep 9: 916-922, 2008.

26. Ko MH and Puglielli L: Two endoplasmic reticulum (ER)/ER Golgi intermediate compartment-based lysine acetyltransferases post-translationally regulate BACE1 levels. J Biol Chem 284: 2482-2492, 2009.

27. Liu M, Wu G, Baysarowich J, et al: PCSK9 is not involved in the degradation of LDL receptors and BACE1 in the adult mouse brain. J Lipid Res 51: 2611-2618, 2010.

28. Shibata N, Ohnuma T, Higashi S, et al: No genetic association between PCSK9 polymorphisms and Alzheimer's disease and plasma cholesterol level in Japanese patients. Psychiatr Genet 15: 239, 2005.

29. Reynolds CA, Hong MG, Eriksson UK, et al: Analysis of lipid pathway genes indicates association of sequence variation near SREBF1/TOM1L2/ATPAF2 with dementia risk. Hum Mol Genet 19: 2068-2078, 2010.

30. Mattson MP and Camandola S: NF- $\kappa B$ in neuronal plasticity and neurodegenerative disorders. J Clin Invest 107: 247-254, 2001.

31. Sola S, Aranha MM and Rodrigues CM: Driving apoptosis-relevant proteins toward neural differentiation. Mol Neurobiol 46: 316-331, 2012.

32. Culmsee $C$ and Plesnila N: Targeting Bid to prevent programmed cell death in neurons. Biochem Soc Trans 34: 1334-1340, 2006.

33. Wong HK, Fricker M, Wyttenbach A, et al: Mutually exclusive subsets of $\mathrm{BH} 3$-only proteins are activated by the p53 and c-Jun N-terminal kinase/c-Jun signaling pathways during cortical neuron apoptosis induced by arsenite. Mol Cell Biol 25: 8732-8747, 2005.

34. Lindsten T, Zong WX and Thompson CB: Defining the role of the Bcl-2 family of proteins in the nervous system. Neuroscientist 11: 10-15, 2005.

35. Almeida A: Genetic determinants of neuronal vulnerability to apoptosis. Cell Mol Life Sci 70: 71-88, 2013.

36. Gotz R, Karch C, Digby MR, Troppmair J, Rapp UR and Sendtner M: The neuronal apoptosis inhibitory protein suppresses neuronal differentiation and apoptosis in PC12 cells. Hum Mol Genet 9: 2479-2489, 2000.

37. Baratchi S, Kanwar RK and Kanwar JR: Survivin: a target from brain cancer to neurodegenerative disease. Crit Rev Biochem Mol Biol 45: 535-554, 2010.

38. Dhandapani KM and Brann DW: Transforming growth factor- $\beta$ : a neuroprotective factor in cerebral ischemia. Cell Biochem Biophys 39: 13-22, 2003.

39. Singh MH, Brooke SM, Zemlyak I and Sapolsky RM: Evidence for caspase effects on release of cytochrome $c$ and AIF in a model of ischemia in cortical neurons. Neurosci Lett 469: 179-183, 2010.

40. Chiou SH, Chen SJ, Peng CH, et al: Fluoxetine up-regulates expression of cellular FLICE-inhibitory protein and inhibits LPS-induced apoptosis in hippocampus-derived neural stem cell. Biochem Biophys Res Commun 343: 391-400, 2006. 
41. Broughton BR, Reutens DC and Sobey CG: Apoptotic mechanisms after cerebral ischemia. Stroke 40: e331-e339, 2009.

42. Blaise S, Kneib M, Rousseau A, et al: In vivo evidence that TRAF4 is required for central nervous system myelin homeostasis. PLoS One 7: e30917, 2012.

43. LeBlanc AC: The role of apoptotic pathways in Alzheimer's disease neurodegeneration and cell death. Curr Alzheimer Res 2: 389-402, 2005.

44. Joseph D'Ercole A and Ye P: Expanding the mind: insulin-like growth factor I and brain development. Endocrinology 149 5958-5962, 2008.

45. McCarthy MJ, Rubin LL and Philpott KL: Involvement of caspases in sympathetic neuron apoptosis. J Cell Sci 110 (Pt 18): 2165-2173, 1997.
46. Ludwig-Galezowska AH, Flanagan L and Rehm M: Apoptosis repressor with caspase recruitment domain, a multifunctional modulator of cell death. J Cell Mol Med 15: 1044-1053, 2011

47. Gora-Kupilas K and Josko J: The neuroprotective function of vascular endothelial grow th factor (VEGF). Folia Neuropathol 43: 31-39, 2005

48. Krantic S,Mechawar N,Reix S and Quirion R: Apoptosis-inducing factor: a matter of neuron life and death. Prog Neurobiol 81: 179-196, 2007. 